\title{
OMEGA-POLISH SPACES AND MEASURABLE SELECTIONS
}

\author{
LE VAN TU
}

(Received 4 November 1975; revised 31 March 1976)

\begin{abstract}
In this paper, the author introduces the notion of $\Omega$-Polish spaces (which includes the Polish spaces and a large class of Banach spaces) and extends Castaing's selection theorem (1966) for closed-valued measurable thin multifunctions from a measurable space into an $\Omega$-Polish space. He also extends Robertson's theorem (1974) in the same way.
\end{abstract}

\section{Introduction}

In the selection problem, we are given a function $F$ from a measurable space $S$ to the set of all non-empty subsets of a topological space $X$ such that the values of $F$ are closed and $F$ is measurable in the sense that for every closed subset $A$ of $X$, the set $\{t \in S: F(t) \cap A \neq \varnothing\}$ is measurable. The problem is to find conditions so that $F$ admits a measurable selector $f$, i.e. a measurable function from $S$ to $X$ satisfying $f(t) \in F(t)$ for every $t \in S$.

Kuratowski and Ryll-Nardzewski (1965) and Castaing (1966) have proved the existence of a measurable selector for $F$ when $X$ is a Polish space (i.e. a complete separable metric space). Himmelberg and Van Vleck (1969) proved this result for the case where $X$ is a Lusin space (i.e. a separable metric space which is the image of a Polish space under a continuous one-to-one mapping), and recently, Robertson (1974) went further, proving this result for the case where $X$ is merely the continuous image of a Polish space.

In this paper we observe that in Castaing's Theorem (1966; Theorem 3) the condition that $X$ is Polish can be relaxed by supposing that $X$ is coverable by a collection of Polish pieces provided that each $F(t)$ does not meet too many of them. This will be shown in Section 3 (Theorem 3). Also in this section we shall extend Robertson's result in the same way (Lemma 1 and Theorems 4,5). 
In section 2 the basic notions of $\Omega$-Polish coverings and $\Omega$-Polish spaces are introduced, and we study in particular how a Banach space (or more generally a linear subspace of a Banach space) can be given a fit $\Omega$-Polish covering (Theorems 1, 2 and Corollaries 1,2).

Throughout this paper we will assume the continuum hypothesis, and denote by $\Omega$ the first uncountable ordinal (see Choquet (1969), page 11).

I should like to express my thanks to Professor A. P. Robertson for many helpful discussions and suggestions.

\section{2. $\mathbf{\Omega}$-Polish spaces}

Definition 1. Let $X$ be a topological space and $\mathscr{S}$ be a family of subsets of $X$. We say that $\mathscr{S}$ is an $\Omega$-Polish covering of $X$ iff $\mathscr{S}$ has cardinal at most $\Omega$, the members of $\mathscr{S}$ are closed in $X$, are Polish spaces with the induced topology and their union is $X$. The pair $(X, \mathscr{P})$ is called an $\Omega$-Polish space.

The same topological space $X$ may have many $\Omega$-Polish coverings. Indeed, since a Polish space has at most $\Omega$ points, every $\Omega$-Polish space has at most $\Omega$ points. Thus a $T_{1}$-space with at most $\Omega$ points always admits the trivial $\Omega$-Polish covering consisting of $\Omega$ one-point sets. At the other extreme a Polish space has a best covering (by one Polish piece).

As we shall see in the next section, selection theorems can be proved for multifunctions $F$ with the property that each $F(t)$ is closed and meets at most countably many of the Polish pieces in the $\Omega$-Polish covering. Thus the scope of the theorems in Section 3 is greatest if the Polish pieces are as large as possible and overlap as little as possible, and if there are plenty of closed sets meeting at most countably many of these pieces. The extreme case, when every closed set has this property, is the subject of Definition 4 at the end of this paper. Meanwhile, in the examples given below of $\Omega$-Polish coverings, we check that there are reasonably-large closed subsets meeting at most countably many Polish pieces. To facilitate this we make the following definition.

Definition 2. An $\Omega$-Polish covering $\mathscr{S}$ of a topological space $X$ is called fit iff there is at least one uncountable closed subset of $X$ meeting at most countably many members of $\mathscr{S}$.

Example 1. Let $X$ be the topological sum of $\Omega$-many uncountable Polish spaces. Then clearly $X$ has a fit $\Omega$-Polish covering, although it is not Polish.

Example 2. Let $X=l^{\infty}$ be the space of all bounded real sequences $x=\left(x_{n}\right)$ indexed by means of the positive integers, endowed with the supremum norm: 


$$
\|x\|=\sup _{n}\left|x_{n}\right|
$$

We know that $l^{\infty}$ is a non-separable Banach space. For each bounded sequence $\alpha=\left(\alpha_{n}\right)$, where $\alpha_{n} \in 2^{-n+1} Z$ ( $Z$ is the set of all integers), let

$$
X_{\alpha}=\left\{x \in X:\left|x_{n}-\alpha_{n}\right| \leqq 2^{-n} \text { for all } n\right\} .
$$

Then each $X_{\alpha}$ is a Polish space. In fact, it is closed since

$$
X_{\alpha}=\bigcap_{n=1}^{\infty}\left\{x \in X:\left|x_{n}-\alpha_{n}\right| \leqq 2^{-n}\right\}
$$

and it is separable since the set of sequences of the form $\left(\alpha_{n}+r_{n}\right)$, where the $r_{n}$ 's are rational, all but a finite number of them are 0 and $\left|r_{n}\right| \leqq 2^{-n}$ for every $n$, is dense in $X_{\alpha}$.

It is clear that

$$
X=\bigcup_{\alpha} X_{\alpha}
$$

where $\alpha$ runs over a subset of $\prod_{n=0}^{\infty} 2^{-n} Z$ which has cardinal $\Omega$. It can also be seen that there are plenty of uncountable closed sets meeting only countably many of the $X_{\alpha}$ 's, e.g. $A=\left\{x \in X:\left|x_{n}\right| \leqq 2^{-n-1}\right.$ for all $\left.n\right\}$ meets only one. Thus the family $\left\{X_{\alpha}\right\}$ is a fit $\Omega$-Polish covering of $X$.

More generally we have the following theorem.

THEOREM 1. Let $X$ be a Banach space possessing a biorthogonal system $\left(e_{n}, f_{n}\right)$ (i.e. $\left\{e_{n}\right\} \subset X,\left\{f_{n}\right\} \subset X^{*}$ and $f_{i}\left(e_{j}\right)=1$ if $i=j$ and zero otherwise) such that $\left\{f_{n}\right\}$ is total on $X$ (i.e. $f_{n}(x)=0$ for all $n$ implies $x=0$ ). Then $X$ has a fit $\Omega$-Polish covering.

Proof. We may suppose that $\sup _{n}\left\|e_{n}\right\|<\infty$ without loss of generality. Let $E$ be the closed linear subspace spanned by $\left\{e_{n}\right\}$. Clearly $E$ is complete and separable. Take any sequence of positive real numbers $\left\{\varepsilon_{n}\right\}$ such that $\sum_{1}^{\infty} \varepsilon_{n}<\infty$ and let

$$
F=\left\{x \in X:\left|f_{n}(x)\right| \leqq \varepsilon_{n} \text { for all } n\right\} .
$$

Then $F$ is a closed subset of $E$; hence $F$ is a Polish space. Now let

$$
I=\left\{x \in X: f_{n}(x) \in 2 \varepsilon_{n} Z \text { for all } n\right\},
$$

where $Z$ is the set of all integers. Certainly $I$ contains all the elements $\sum_{n=1}^{m} 2 k_{n} \varepsilon_{n} e_{n}$, where $k_{n} \in Z$ for every $n$. Furthermore $I$ has cardinal at most $\Omega$, since the map $x \rightarrow\left\{f_{n}(x)\right\}$ from $I$ into $\Pi_{n=1}^{\infty} \varepsilon_{n} Z$, is one-to-one by the totality of $\left\{f_{n}\right\}$. 
We now prove that

$$
X=F+I \text {. }
$$

In fact, let $x \in X$; then there exists a sequence of integers $\left\{k_{n}\right\}$ such that $\left|f_{n}(x)-2 k_{n} \varepsilon_{n}\right| \leqq \varepsilon_{n}$ for every $n$. This implies that the series $\Sigma_{1}^{\infty}\left|f_{n}(x)-2 k_{n} \varepsilon_{n}\right|$ is convergent; hence the series $\Sigma_{1}^{\infty}\left(f_{n}(x)-2 k_{n} \varepsilon_{n}\right) e_{n}$ converges to some $y \in E$ and $f_{n}(y)=f_{n}(x)-2 k_{n} \varepsilon_{n}$, for every $n$. Thus $y \in F$ and $f_{n}(x-y)=2 k_{n} \varepsilon_{n}$ for every $n$, i.e. $x-y \in I$. Therefore $x \in F+I$.

Again, it is easy to see that there are plenty of uncountable closed sets meeting only countably many $F+x$ with $x \in I$; e.g. $A=\left\{x=\lambda e_{1}:|\lambda| \leqq \varepsilon_{1} / 2\right\}$ meets $F$ only. Thus the family $\{F+x: x \in I\}$ is a fit $\Omega$-Polish covering of $X$. This completes the proof.

Corollary 1. Let $X$ be a Banach space with a basis. Then $X^{*}$ has a fit $\Omega$-Polish covering.

Notice that the condition that $X$ has a basis is equivalent to that $X^{*}$ has a $w^{*}$-Schauder basis, i.e. a basis such that its associated coefficient functionals are weak*-continuous (Singer (1970), Theorem 14.1, page 155).

Proof. Let $\left\{e_{n}\right\}$ be a basis of $X$ and let $\left\{f_{n}\right\}$ be its associated coefficient functionals. Then the functions $f_{n}$ are continuous, i.e. $f_{n} \in X^{*}$ (Singer (1970), Theorem 3.1, page 20).

For each $n$, let $\phi_{n}$ be the image of $e_{n}$ by the canonical injection of $X$ into $X^{* *}$. Then clearly the system $\left(f_{n}, \phi_{n}\right)$ is biorthogonal. Furthermore, the sequence $\left\{\phi_{n}\right\}$ is total on $X^{*}$, since if $\phi_{n}(g)=0$ for every $n$, then $g\left(e_{n}\right)=0$ for every $n$, and therefore $g=0$. Thus Theorem 1 can be applied for $X^{*}$. This completes the proof.

The $\Omega$-Polish spaces presented above are complete. We may generalize Theorem 1 to obtain non-complete $\Omega$-Polish spaces as follows.

TheOREM 2. Let $X$ be a Banach space that has a biorthogonal system $\left(e_{n}, f_{n}\right)$ satisfying the conditions of Theorem 1. Also let $\left\{\varepsilon_{n}\right\}$ be a sequence of positive real numbers such that $\sum_{1}^{\infty} \varepsilon_{n}<\infty$. Then every linear subspace $Y$ of $X$, containing all the elements $x$ such that $\left|f_{n}(x)\right| \leqq \varepsilon_{n}$ for every $n$, has a fit $\Omega$-Polish covering.

Note that the subspace $Y$ is not necessarily closed. For example, $\left\{x \in X: \Sigma_{1}^{\infty} f_{n}(x) e_{n}\right.$ converges $\}$ is a linear subspace of $X$ with the required property, and it is not necessarily closed.

Proof. The argument is entirely the same as in the proof of Theorem 1 , except that we put 


$$
J=\left\{x \in Y: f_{n}(x) \in 2 \varepsilon_{n} Z \text { for all } n\right\}
$$

and we have

$$
Y=F+J
$$

The following corollary is an evident consequence of Theorem 2 .

Corollary 2. Let $X$ be a Banach space with a basis $\left\{e_{n}\right\}$. Also let $\left\{\varepsilon_{n}\right\}$ be a sequence of positive real numbers such that $\Sigma_{1}^{\infty} \varepsilon_{n}<\infty$. Then every linear subspace of $X^{*}$; containing all the elements $f$ such that $\left|f\left(e_{n}\right)\right| \leqq \varepsilon_{n}$ for every $n$, has a fit $\Omega$-Polish covering.

Proof. With the notations as in the proof of Corollary 1 , we have

$$
\left\{f \in X^{*}:\left|f\left(e_{n}\right)\right| \leqq \varepsilon_{n}, \forall n\right\}=\left\{f \in X^{*}:\left|\phi_{n}(f)\right| \leqq \varepsilon_{n}, \forall n\right\} .
$$

Thus Theorem 2 can be applied.

\section{Selection theorems}

Let $S$ be a measurable space (i.e. a set with a $\sigma$-algebra $\mathcal{M}$ of subsets) and let $X$ be a topological space. Also let $F$ be a closed-valued multifunction from $S$ to $X$, that is, $F$ assigns to each $t \in S$ a non-empty closed set $F(t) \subseteq X$. For any subset $A$ of $X$, let

$$
F^{-1}(A)=\{t \in S: F(t) \cap A \neq \varnothing\} .
$$

We say that $F$ is measurable iff $F^{-1}(A) \in \mathcal{M}$ for every closed subset $A$ of $X$. A point-function $f$ from $S$ to $X$ is called a measurable selector of $F$ iff $f(t) \in F(t)$ for every $t \in S$ and $f^{-1}(A) \in \mathcal{M}$ for each closed set $A$ of $X$.

Kuratowski and Ryll-Nardzewski (1965) and Castaing (1966) have proved the following theorem.

Theorem A. Let $S$ be a measurable space and let $X$ be a Polish space. Then every closed-valued measurable multifunction from $S$ to $X$ has a measurable selector.

Now let $\left(X,\left(X_{\alpha}\right)\right)$ be an $\Omega$-Polish space (where each $X_{\alpha}$ is a Polish space and closed in $X$ ). We define a thin multifunction from $S$ to $X$ as follows.

Definition 3. A multifunction $F$ from $S$ to $X$ is called thin iff for any proper closed subset $A$ of $X$ the set $F\left(F^{-1}(A)\right)$ meets at most countably many members of the family $\left\{A \cap X_{\alpha}: \alpha<\Omega\right\}$.

Certainly if $F(S)=\cup\{F(t): t \in S\}$ meets only a countable number of the $X_{\alpha}$ 's, then $F$ is thin. In the following example, we shall see that $F$ may be thin and yet $F(S)$ may meet an uncountable number of the $X_{\alpha}$ 's. 
ExAmple 3. Let $E=\{(x, 0): 0 \leqq x \leqq 1\}, E^{\prime}=\{(\alpha, 0): 1 \leqq \alpha \leqq 2\}$ and for each $\alpha \in[1,2]$, let $T_{\alpha}=\{(\alpha, y): 0 \leqq y \leqq 1\}$ if $\alpha$ is rational and $T_{\alpha}=\{(\alpha, 0)\}$ if $\alpha$ is irrational. Now let

$$
X=E \cup\left(\cup\left\{T_{\alpha}: 1 \leqq \alpha \leqq 2\right\}\right) .
$$

We define a topology for $X$ by setting that a subset $G$ of $X$ is open iff either $G$ is empty or $G$ satisfies the following conditions:

(i) $G \cap E$ is open in $E$ with respect to the usual topology of $E$,

(ii) for every $\alpha, G \cap T_{\alpha}$ is open in $T_{\alpha}$ with respect to the usual topology of $T_{\alpha}$,

(iii) $E^{\prime} \backslash G$ is at most countable.

It can be seen that $X$ is not Polish, in fact not separable, since if $\left\{q_{n}\right\}$ is a sequence of points of $X$, then $G=E^{\prime} \backslash\left(\left\{q_{n}\right\} \cup\{(\alpha, 0)\right.$ : $\alpha$ rational, $\left.1 \leqq \alpha \leqq 2\}\right)$ is a non-empty open subset of $X$ that does not meet $\left\{q_{n}\right\}$.

For every $\alpha \in[1,2]$, let $X_{\alpha}=E \cup T_{\alpha}$. Clearly each $X_{\alpha}$ is closed in $X$. Furthermore, the induced topology of $X_{\alpha}$ coincides with its usual topology; hence each $X_{\alpha}$ is a Polish space. Thus the family $\left\{X_{\alpha}: 1 \leqq \alpha \leqq 2\right\}$ forms an $\Omega$-Polish covering for $X$. Now let $F$ be a multifunction from some space $S$ to $X$ such that $F(S)=\cup\left\{T_{\alpha}: 1 \leqq \alpha \leqq 2\right\}$. Clearly $F(S)$ meets all the $X_{\alpha}$ 's. Let $A$ be any proper closed subset of $X$, then $X \backslash A$ is a non-empty open set of $X$; hence $A \cap E^{\prime}$ is at most countable. Therefore $A$ meets at most a countable number of the $T_{\alpha}$ 's.

REMARK. In the above example, we adjoined the segment $E$ to avoid the particular case in which $X$ is a thin $\Omega$-Polish space which we shall define later (Definition 4). Also we adjoined the segments $T_{\alpha}$ to allow $F$ to have uncountable set-values.

The following is an extension of Theorem A.

Theorem 3. Let $S$ be a measurable space and $X$ be an $\Omega$-Polish space. Then every closed-valued measurable thin multifunction $F$ from $S$ to $X$ has $a$ measurable selector.

Proof. Let $\mu$ be the $\sigma$-algebra of subsets of $S$ and let $X=\cup\left\{X_{\alpha}: \alpha<\right.$ $\Omega$, where each $X_{\alpha}$ is a Polish space and closed in $X$. For each $\alpha<\Omega$, put

$$
S_{\alpha}=F^{-1}\left(X_{\alpha}\right) \backslash \cup\left\{F^{-1}\left(X_{\beta}\right): \beta<\alpha\right\} .
$$

Clearly $S_{\alpha} \in \mathcal{M}$, the $S_{\alpha}$ 's are pairwise disjoint and their union is $S$.

Now let $\mathcal{M}_{\alpha}=\left\{E \cap S_{\alpha}: E \in \mathcal{M}\right\}$; then $\mathcal{M}_{\alpha}$ is a $\sigma$-algebra of subsets of $S_{\alpha}$ and since $S_{\alpha} \in \mathcal{M}, \mathcal{M}_{\alpha} \subseteq \mathcal{M}$. For each $\alpha$, define the multifunction $F_{\alpha}$ from $S_{\alpha}$ to $X_{\alpha}$ by 


$$
F_{\alpha}(t)=F(t) \cap X_{\alpha}, \quad t \in S_{\alpha} .
$$

Clearly $F_{\alpha}(t)$ is non-empty and closed; furthermore, $F_{\alpha}$ is $\mathcal{M}_{\alpha}$-measurable. In fact, for any closed $A$ in $X_{\alpha}, A$ is also closed in $X$ and we have

$$
F_{\alpha}^{-1}(A)=F^{-1}(A) \cap S_{\alpha} \text {. }
$$

Therefore, by Theorem A, $F_{\alpha}$ has a measurable selector $f_{\alpha}: S_{\alpha} \rightarrow X_{\alpha}$. Let us define $f: S \rightarrow X$ by

$$
f(t)=f_{\alpha}(t) \text { if } t \in S_{\alpha} .
$$

Certainly $f$ is a selector for $F$; we shall prove that $f$ is measurable by considering a proper closed subset $A$ of $X$. Then

$$
f^{-1}(A)=\cup\left\{f_{\alpha}^{-1}\left(A \cap X_{\alpha}\right): \alpha<\Omega\right\},
$$

where each $f_{\alpha}^{-1}\left(A \cap X_{\alpha}\right) \in \mathcal{M}_{\alpha} \subseteq \mathcal{M}$. We claim that the sets $f_{\alpha}^{-1}\left(A \cap X_{\alpha}\right)$ are empty except a countable number of them. In fact, since $F$ is a thin multifunction, there exists $\alpha_{0}<\Omega$ such that $F\left(F^{-1}(A)\right)$ does not meet $A \cap X_{\alpha}$ for every $\alpha>\alpha_{0}$. Now if, for some $t, f_{\alpha}(t) \in A \cap X_{\alpha}$, then $F_{\alpha}(t)$ and so $F(t)$ would meet $A \cap X_{\alpha}$ and then $F\left(F^{-1}(A)\right) \cap A \cap X_{\alpha}$ would not be empty. But this cannot happen for $\alpha>\alpha_{0}$ and so $f_{\alpha}^{-1}\left(A \cap X_{\alpha}\right)=\varnothing$ for all $\alpha>\alpha_{0}$. Therefore $f^{-1}(A)$ is measurable. This completes the proof.

Before going on we introduce the conception of Souslin operation which can be found in Rogers (1970). Let $\sigma=\left(i_{1}, i_{2}, \cdots\right)$ be an infinite sequence of positive integers; we shall use the notation $\sigma \mid n$ to indicate the finite sequence $i_{1}, i_{2}, \cdots, i_{n}$. By a Souslin- $\mathcal{M}$ set we mean a set $A$ of the form

$$
A=\bigcup_{\sigma} \bigcap_{n} A_{\sigma \mid n},
$$

where $\sigma$ runs over $N^{N}, n=1,2,3, \cdots$ and each $A_{\sigma \mid n}$ is a member of $\mathcal{M}$.

We say that the measurable space $S$ (with the $\sigma$-algebra $\mathscr{M}$ ) admits the Souslin operation iff $\mathcal{M}$ contains all Souslin- $\mathcal{M}$ sets.

The following lemma is an extension of Lemma 1 in Robertson (1974) and the proof is just a slight modification of his.

Lemma 1. Let $S$ be a measurable space that admits the Souslin operation and $Y$ be a regular space, the continuous image of an $\Omega$-Polish space $X$ by $\phi$. Also let $F$ be a closed-valued measurable multifunction from $S$ to $Y$. If the multifunction $\phi^{-1} \circ F$ is thin, then $F^{-1}(\phi(A))$ is measurable for every closed set $A$ of $X$.

THEOREM 4. Let $S$ be a measurable space that admits the Souslin operation and $Y$ be a regular space, the continuous image of an $\Omega$-Polish space $X$ by 
$\phi$. Also let $F$ be a closed-valued measurable multifunction from $S$ to $Y$ such that $\phi^{-1} \circ F$ is a thin multifunction. Then $F$ has a measurable selector.

Proof. Let $G=\phi^{-1} \circ F$. Clearly $G$ is closed-valued and thin; furthermore $G$ is measurable, since, for any closed $A$ of $X, G^{-1}(A)=F^{-1}(\phi(A))$ is measurable by Lemma 1 . Hence, by Theorem $3, \mathrm{G}$ has a measurable selector $g$. It follows that $f=\phi \circ g$ is a measurable selector for $F$.

We may also transfer the thinness from the multifunction $F$ to the $\Omega$-Polish space $X$ as in the Definition 4 which follows. However the theorem obtained, Theorem 5, is certainly weaker than Theorem 4 (cf. Example 3).

Definition 4. An $\Omega$-Polish space $(X, \mathscr{P})$ is called thin iff every proper closed subset $A$ of $X$ meets at most countably many members of $\mathscr{S}$.

Again every Polish space is a thin $\Omega$-Polish space. In the Example 3, if we discarded the segment $E$, we would have a thin $\Omega$-Polish space that is not Polish.

THEOREM 5. Let $S$ be a measurable space that admits the Souslin operation and $Y$ be a regular space, the continuous image of a thin $\Omega$-Polish space $X$ by $\phi$. Then every closed-valued measurable multifunction $F$ from $S$ to $Y$ has a measurable selector.

Proof. In this case any multifunction from $S$ to $X$ is thin. Hence Theorem 4 can be applied.

It should be noted that if $F$ is a point-function, then the arguments in the proofs of Lemma 1 and Theorem 4 are still valid if we suppose $Y$ to be Hausdorff instead of being regular. This gives us the following corollary related to the lifting problem (see McShane and Warfield (1967) for other cases).

Corollary 3. Let $S, X, Y$ and $\phi$ be as in Theorem 5, except that $Y$ is assumed to be Hausdorff. Then every point-valued measurable function $f$ from $S$ to $Y$ can be lifted to a measurable function $g$ from $S$ to $X$ (i.e. $f=\phi \circ g$ ).

\section{References}

C. Castaing (1966), 'Quelques problèmes de mesurabilité liés à la théorie de la commande', $C$. $R$. Acad. Sc., Paris, 262, 409-411.

G. Choquet (1969), Lectures on Analysis, Vol. I (W.A. Benj., New York, 1969).

C. J. Himmelberg and F. S. Van Vleck (1969), 'Some selection theorems for measurable functions', Can. J. Math. 21, 394-399.

K. Kuratowski and C. Ryll-Nardzewski (1965), 'A general theorem on selectors', Bull. Acad. Polon. Math., Astr., Phys., 13, 397-403. 
E. J. MeShane and R. B. Warfield Jr., (1967), 'On Filipov's implicit functions lemma', Proc. Amer. Math. Soc., 18, 41-47.

A. P. Robertson (1974), 'On measurable selections', Proc. R. Soc. Edinburgh, 72, 1-7.

C. A. Rogers (1970), Hausdorff measures (Cambridge, Univ. Press, page 44).

I. Singer (1970), Bases in Banach spaces, Vol. I (Springer, New York, 1970).

Murdoch University,

Murdoch, 6153,

Western Australia. 